\title{
Single Piezo-Actuator Rotary-Hammering (SPaRH) Drill
}

\author{
Stewart Sherrit, Lukas Domm, Xiaoqi Bao, \\ Yoseph Bar-Cohen, Zensheu Chang, Mircea Badescu \\ Jet Propulsion Laboratory, California Institute of Technology, Pasadena, CA 91109-8099
}

\begin{abstract}
The search for present or past life in the Universe is one of the most important objectives of NASA's exploration missions. Drills for subsurface sampling of rocks, ice and permafrost are an essential tool for astrobiology studies on other planets. Increasingly, it is recognized that drilling via a combination of rotation and hammering offers an efficient and effective rapid penetration mechanism. The rotation provides an intrinsic method for removal of cuttings from the borehole while the impact and shear forces aid in the fracturing of the penetrated medium. Conventional drills that use a single actuator are based on a complex mechanism with many parts and their use in future mission involves greater risk of failure and/or may require lubrication that can introduce contamination. In this paper, a compact drill is reported that uses a single piezoelectric actuator to produce hammering and rotation of the bit. A horn with asymmetric grooves was designed to impart a longitudinal (hammering) and transverse force (rotation) to a keyed free mass. The drill requires low axial pre-load since the hammering-impacts fracture the rock under the bit kerf and rotate the bit to remove the powdered cuttings while augmenting the rock fracture via shear forces. The vibrations 'fluidize' the powdered cuttings inside the flutes reducing the friction with the auger surface. This action reduces the consumed power and heating of the drilled medium helping to preserve the pristine content of the acquired samples. The drill consists of an actuator that simultaneously impacts and rotates the bit by applying force and torque via a single piezoelectric stack actuator without the need for a gearbox or lever mechanism. This can reduce the development/fabrication cost and complexity. In this paper, the drill mechanism will be described and the test results will be reported and discussed.
\end{abstract}

KEYWORD: Piezoelectric drills, piezoelectric horn transducers, asymmetric grooves structures, rotary motion, ultrasonic impacts, USDC.

\section{INTRODUCTION}

NASA exploration of extraterrestrial bodies increasingly requires compact, low power and low axial preload sampling mechanisms to obtain core samples for in-situ analysis. These samples may help reveal the composition and history of the planets in our solar system, and could also indicate the existence of past or present life. The Ultrasonic/Sonic Driller/Corer (USDC) developed at JPL with is a hammering device, which requires low power, low axial preload (beneficial for use on light drilling platforms), and which can withstand extremes in temperature. One of the problems with pure hammering is that as the teeth become embedded in the sample, the drilling efficiency drops unless the teeth are moved away from the specific foot-print location. This limitation was found in a variety of designs based on the USDC ${ }^{1,2,3}$. By rotating the bit, the teeth are moved to areas that were not fragmented and thus enhancing the rock fracture via shear forces. The shear motion also creates ripping or chiseling action and thus producing larger particles to increase the drilling efficiency and reducing the required power. It has also been demonstrated that unless the cuttings are removed from the hole, the drilling depth for small samplers $(<1 \mathrm{~cm}$. diameter $)$, cannot exceed a few centimeters depth. In some sampler designs the cuttings were removed by pressurized gas flow ${ }^{4}$ or by designing asymmetries in the bit that would cause rotation or oscillations ${ }^{5}$ and finally by rotating the bit with an independent electromagnetic motor ${ }^{6}$. The percussive impact action leads to penetration of the medium by producing a zone of finely crushed rock directly underneath the impacted locations. The fracturing process is enhanced by the shear forces from the rotation and twisting action [Maurer ${ }^{7}$, Bar-Cohen and Zacny ${ }^{8}$ ]. In addition, the rotation can be used to remove the cuttings via an auger on the bit, which can be enhanced by the vibration/fluidization of the cuttings. The powdered cuttings can be induced to travel up the flutes to a slot bailer or to the rock surface. Therefore, a combined hammering and rotary cutting mechanism has many advantages over rotary or hammering alone. The sampler described in this paper is the result of our efforts to combine the rotary and hammering action in a single actuator ${ }^{9,10}$. A similar idea has also been independently suggested by Harkness et al. ${ }^{11,12}$

Ultrasonic motors based on piezoelectric actuation have been available for some time $e^{13,14,15}$. The mode of operation (quasi-static or resonant), type of motion (rotary or linear), and the shape of actuation element (beam, rod, disk, etc.) can 
be used to classify piezoelectric motors. Despite these distinctions, the fundamental principles of solid-state actuation are common to all of these devices. Each of these motor designs uses microscopic material deformations (usually associated with piezoelectric materials), which are amplified through either quasi-static or dynamic/resonant means. In an effort to produce a rotating and hammering bit we examined horn designs which could impart a torque to the free mass that is keyed to the bit to allow independent extension and transmission of the torque to the bit. A schematic of two potential torque generation motions are shown in Figure 1.
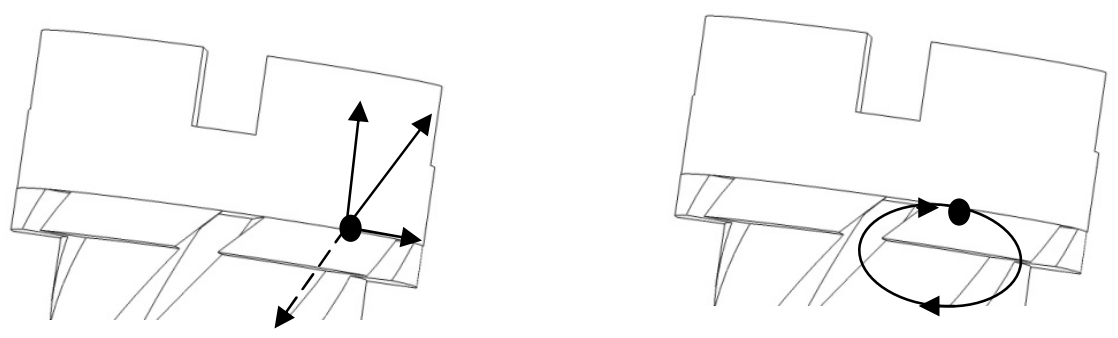

Figure 1: View of the two potential horn motions (helical extension or bending) that can develop an impact and torque at a point (black dot) on the free mass. Black arrow and ellipse show contact. Contact pushes the free mass up and drags it to right in each case. The normal force is much lower on retraction or on the bottom portion of the ellipse. The net force on the left produces a prolonged rotation of the bit as well as an impact. The bending ellipse shown on the right produces a rotation. A mode that rotates in the opposite direction around the ellipse would induce reverse rotation.

The figure on the left shows an off axis extension that produces impact forces that have a component that is perpendicular to the horn's axial axis at an outer radius. The torque generated is, to first order, the time average of the product of the frictional force between the horn and free mass and the radius of contact. The second motion that can impart a torque is the bending motion shown in the right figure. In this case, the surface of the horn follows the ellipse (clockwise) and when the horn contacts the free mass it drags the free mass microscopically. The reverse motion could be produced by a bending action that caused the point (black circle) on the horn to rotate in the opposite direction (counter clockwise). The developed drill enhances the Ultrasonic/Sonic Driller/Corer (USDC) $)^{16,17,18,19}$ since the bit is activated directly by the piezoelectric horn actuator, a single actuator creates the rotation and the hammering action using a keyed free-mass

The USDC was demonstrated to sample powdered cuttings that are very fine with minimal crystallographic structure distortions as was demonstrated in prior NASA related study ${ }^{20}, 45$ weight percent of the powder produced using Chinle Sandstone had a size of less than 5- $\mu$ m measured by a Horiba CAPA-500 particle size distribution analyzer. When sampling basal limestone of the Todilto Formation (Echo Amphitheater, New Mexico) about 55\% of the particle sizes were below $5-\mu \mathrm{m}$. It is expected that these qualities of the USDC will be maintained in the piezoelectric rotary hammer drill since the impact forces are designed to be the same.

The actuator of the sampler is made of a piezoelectric stack that is compressed by a pre-stress bolt between the backing and the horn. In future designs we plan to use a pre-stress flexure to remove the problem of thermal mismatch and pre-stress degradation since the flexure stiffness can be designed to be $>20$ times less than the PZT stack to accommodate CTE differences ${ }^{21}$. The backing is intended to transfer the generated mechanical vibrations towards the horn direction. In order to generate rotation, the horn will be configured with asymmetrically helical or angled slots (see Figure 2) and upon impacting the bit it will introduce longitudinal vibration along the axis and a tangential force and twisting action that will rotate the bit. In addition, bending moments at the horn tip at other frequencies can potentially be used to produce only rotation and little hammering. The longitudinal component of the vibrations of the stack introduces percussion impulses between the bit and the rock to fracture it when the ultimate strain is exceeded under the bit. This novel actuator ${ }^{9}$ is a hybrid between a horn for power ultrasonics using vibratory motion as in the USDC design and an ultrasonic motor configuration that is known as the Kumada piezo-motor ${ }^{13,22 .}$ This motor configuration has been documented to have a high efficiency of $80 \%$, relatively high torque density of $8.8 \mathrm{Nm} / \mathrm{Kg}^{13,22}$. A recent prototype built in the NDEAA lab and shown Figures 5 and 6 demonstrated drilling in limestone at a rate of $0.8 \mathrm{~cm}$ per minute and rotation speeds of 200 RPM. The original horn concept had a helical slot configuration (as shown in Figure 2) which applies rotation forces that turn the bit (acting like a rotor) onto which it is pressed but is also subjected to longitudinal 
vibrations. In order to develop a feasible design we started with the initial concept and iterated design parameters such as the overall length, horn cross section and slot angles.

\section{MODELLING}

The methods used in modeling various horn designs, as well as ease of fabrication and testing put limits on the models we wanted to solve. The initial step of the analysis was to design a horn that had a resonance frequency in a reasonable range and the mounting flange at a nodal plane. Before performing finite element analysis, the horn designs were adjusted using a Mason's Equivalent Circuit model in order to optimize their individual performance and to align the neutral plane. The variety of horn designs that were considered reflects the variety of potential modes available for producing rotation.

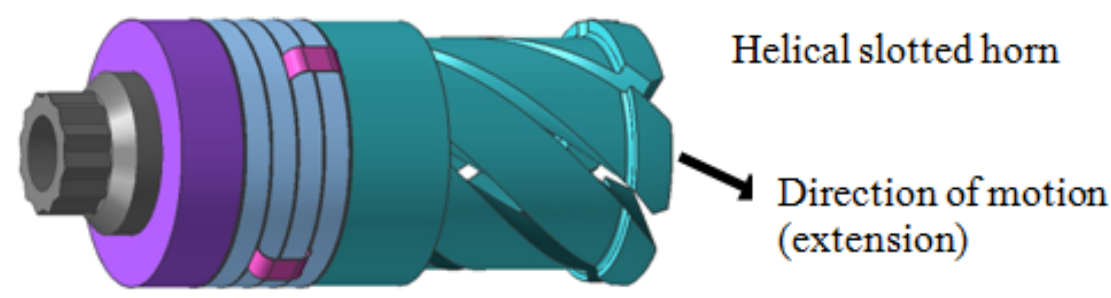

Figure 2: A view of a piezoelectric actuator with a slotted helical horn that simultaneously generates longitudinaltwisting modes to hammer and rotate the bit. This horn actuator has a pre-stress bolt.

\section{A. 1D Network Model}

A Mason's Equivalent Circuit models a piezoelectric actuator as a one-dimensional (1D) network model ${ }^{1}$. The model uses the piezoelectric material constants and the mechanical/acoustic properties of the transducer horn and backing materials and the dielectric properties of the piezoelectric material to determine the stress and displacement at different positions along the actuator. The model was adapted into a MATLAB program for rapid execution. Mason's Equivalent Circuit was used primarily for determining tip extensional displacement, the electromechanical coupling coefficient, and the neutral plane location by quickly changing horn dimensions. By changing the length of the horn or the backing section, the neutral plane could be adjusted before the model was analyzed in Finite Element Analysis software. An example of the displacement at the horn tip and mounting flange location is shown in Figure 3 for the horn shown in Figure 6 . The displacement of the mounting flange has been minimized such that there is a neutral plane at this location. Note that the spike in tip displacement is located at the first extensional mode of the transducer. The second spike represents the second extensional mode, at which the neutral plane is no longer in alignment with the mounting flange, indicated by the high flange displacement.

Once a horn design had been adjusted using the Mason's Equivalent Circuit, the CAD model was analyzed in ANSYS Finite Element software to observe the horn tip motion in both a modal and harmonic analysis. ANSYS Workbench was used as a preliminary test of the general shape of the horn's motion at resonance, which was between $10-30 \mathrm{kHz}$ for most horns examined. The ANSYS Workbench harmonic analysis modeled the piezoelectric effect simply as an arbitrary alternating pressure between the piezoelectric ceramics. This method revealed the general shape of a horn's motion. If the horn appeared to produce rotation at the tip, the design was handled by Finite Element Modal and Harmonic Analyses in ANSYS Multiphysics.

The ANSYS Multiphysics Harmonic Analysis was able to directly model the piezoelectric effect with an applied voltage, and yielded not only the shape of the horn's motion, but the predicted horn tip displacement and rotation. The ANSYS Multiphysics Harmonic Analysis provided the most accurate and complete prediction of a horn's performance. Mason's Equivalent Circuit and ANSYS Workbench were useful only in that each horn could be analyzed rapidly in order to weed out poor designs such that we could concentrate on a complete Finite Element analysis for those horns that appeared promising. 


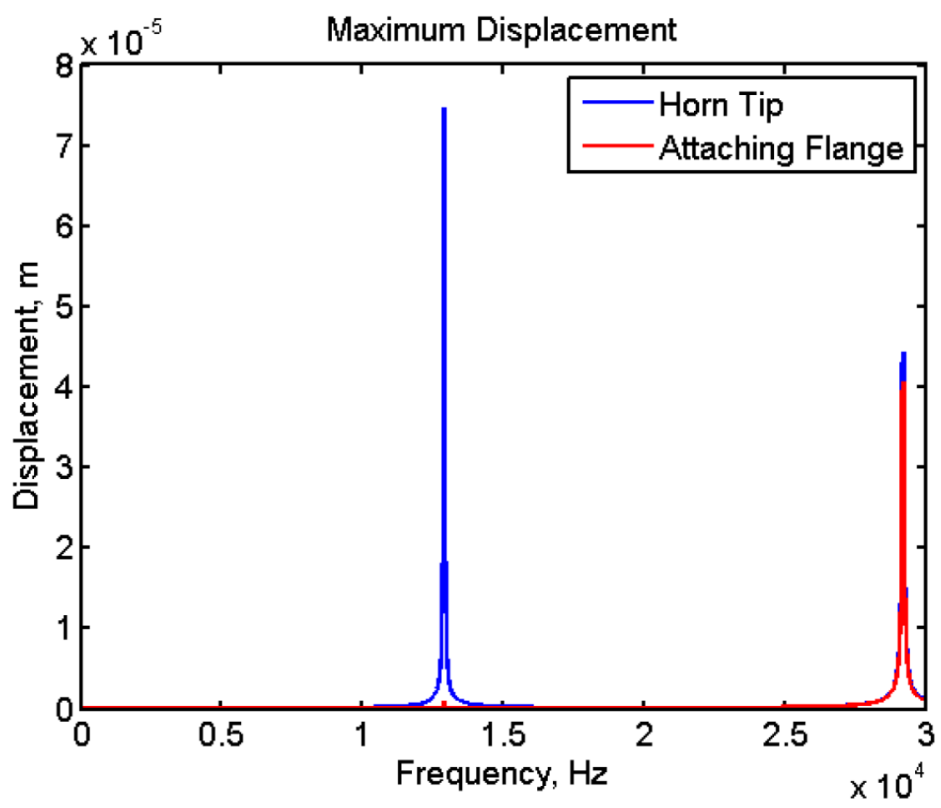

Figure 3: The maximum displacement prediction from Mason's Equivalent Circuit. The program predicts the horn tip and flange maximum displacement over a range of frequencies. The peaks represent first and second extensional modes.

\section{B. Modeling Results and Chosen Design}

Of the many horn designs initially conceived and modeled, only few demonstrated simultaneous rotation and extension at the horn tip. The Modal Analysis finite element results of two of the models that failed to produce sufficient rotation and extension at the horn tip are shown in Figure 4.
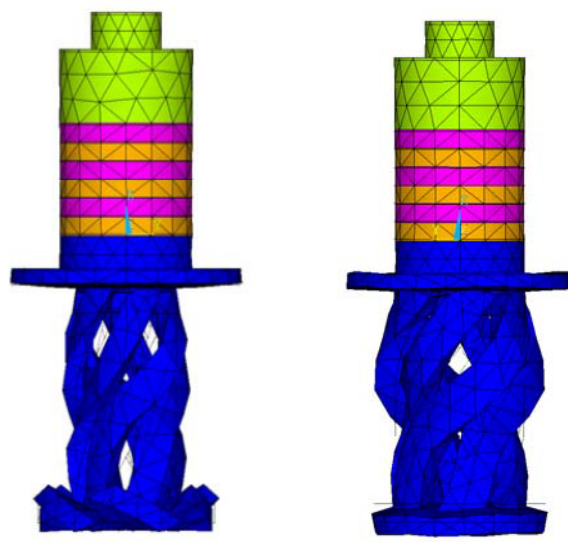

Figure 4: Finite element models of two rejected horn designs. The purple and gold areas (thin disks) represent the piezoelectric ceramics. These horns do not display sufficient rotation and extension at the horn tip at resonance.

Most of the horns that were initially modeled were essentially a hollow tube with angled or helical cuts, as seen in Figure 4 above. To the left in Figure 4, the cuts extend through the horn so as to create individual horn 'tines'. The behavior of these individual tines when excited at resonance did not produce tip rotation, and the motion appeared somewhat arbitrary. It was thought that connecting the tines at the horn tip (as demonstrated by the horn to the right in Figure 4) would promote a more uniform extension/rotation at the horn tip. Tip extension was increased, but rotation was still absent at the tip. The final horn selected for fabrication used a webbed cross section design, such that the cuts do not extend entirely through the horn, but leave webbing around a center hole. The webbed cross section of a horn is 
shown in left of Figure 5. This webbing seemed to provide a means to keep the horn tine motion uniform while still giving the horn torsional flexibility, since the webbing connection is toward the center of the horn where resistance to torsion would be lowest. The final design selected for fabrication demonstrated simultaneous extension and rotation at the horn tip. The Finite Element model of the chosen design is shown in Figure 5.
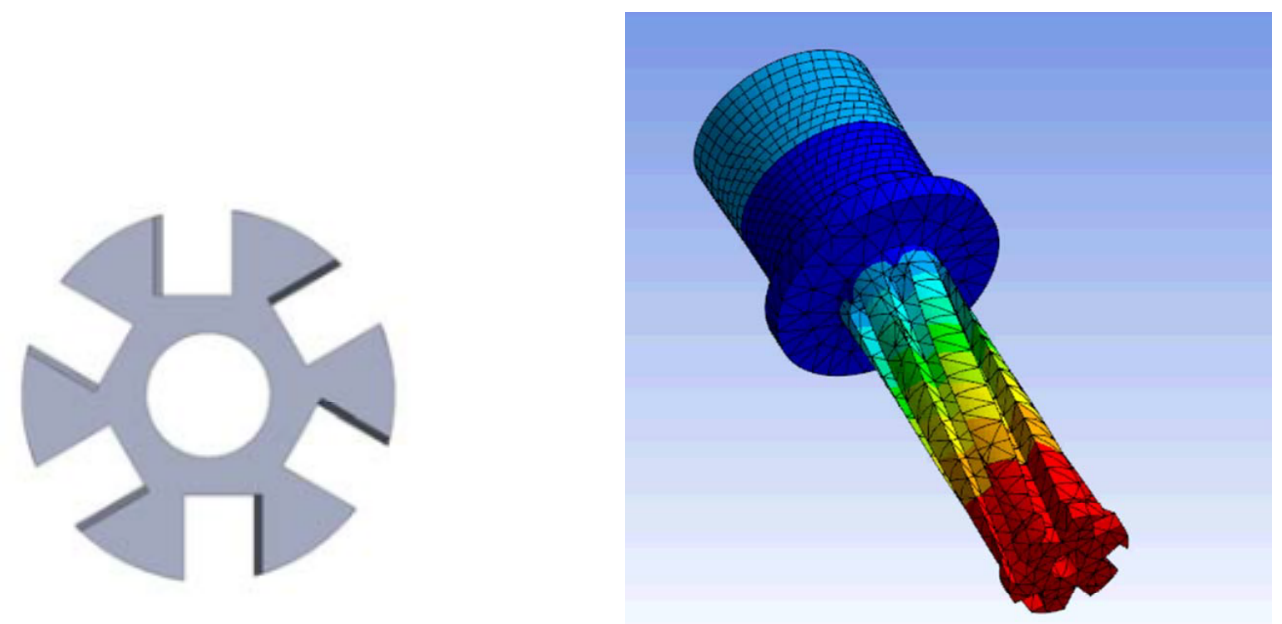

Figure 5. The cross section of webbed horn. Angled cuts extend only partway through the horn to leave a webbing ring connecting individual horn tines. A Finite Element Modal analysis of selected transducer design. Dark blue represents areas of minimal motion at resonance, while red as see at the tip represents areas of maximum displacement. The angled cuts in the horn cause the tip to rotate counter-clockwise as it extends in resonance.

Table 1. ANSYS predicted performance of chosen transducer design at $1 \mathrm{~W}$ input power.

\begin{tabular}{|l|l|l|l|c|}
\hline $\begin{array}{l}\text { Frequency } \\
\text { (First axial } \\
\text { mode) }\end{array}$ & $\begin{array}{l}\text { Coupling } \\
\text { Coefficient }\end{array}$ & $\begin{array}{l}\text { Tip } \\
\text { Displacement, } \\
\boldsymbol{\mu m}\end{array}$ & $\begin{array}{l}\text { Tip Rotation, } \\
\text { rad }\end{array}$ & Voltage, V \\
\hline 12217 & 0.08 & 1.75 & $4.40 \mathrm{E}-5$ & 27.0 \\
\hline
\end{tabular}

The above design demonstrated simultaneous extension and rotation at the horn tip. The tip tends to rotate counterclockwise (looking from the horn tip) as it extends in resonance as a result of the angled cuts in the horn. The transducer performance for 1 Watt input power (actual drive power would be between 50 to 100 Watts) as predicted by ANSYS Harmonic Analysis is shown in the table below. It should be noted that $\mathrm{k}=0.08$ is a low coupling coefficient. Typical horns for the USDC demonstrate a coupling of about $\mathrm{k}=0.2$ to 0.4 . Due to the proper tip movement and time constraints, this low value was accepted. The Piezoelectric Rotary Hammer Drill prototype is primarily designed to verify that a single piezoelectric actuator can produce both rotation and hammering in a drill string, and while a high coupling coefficient is desirable, it does not play a critical role in achieving this main goal of the research. Future designs should attempt to maximize this parameter.

\section{FABRICATION AND RESULTS}

A Piezoelectric Rotary Hammer Drill prototype was fabricated and tested. The transducer was analyzed using an impedance analyzer in order to obtain the actual resonant frequency and electromechanical coupling coefficient. The horn tip displacement and rotation were also measured using a Fotonic Sensor. Measurements of the horn tip rotation were used to predict the rotation speed that will be produced in the drill bit, in order to begin to characterize the rotation produced by the drill. Finally, the assembled Piezoelectric Rotary Hammer Drill was tested while drilling in limestone. 


\section{A. Transducer Performance}

Once the modeling was complete and a design had been chosen, an initial prototype of the Piezoelectric Rotary Hammer Drill transducer was fabricated. A photograph of the transducer is shown in the Figure 6 along with its admittance spectra below.
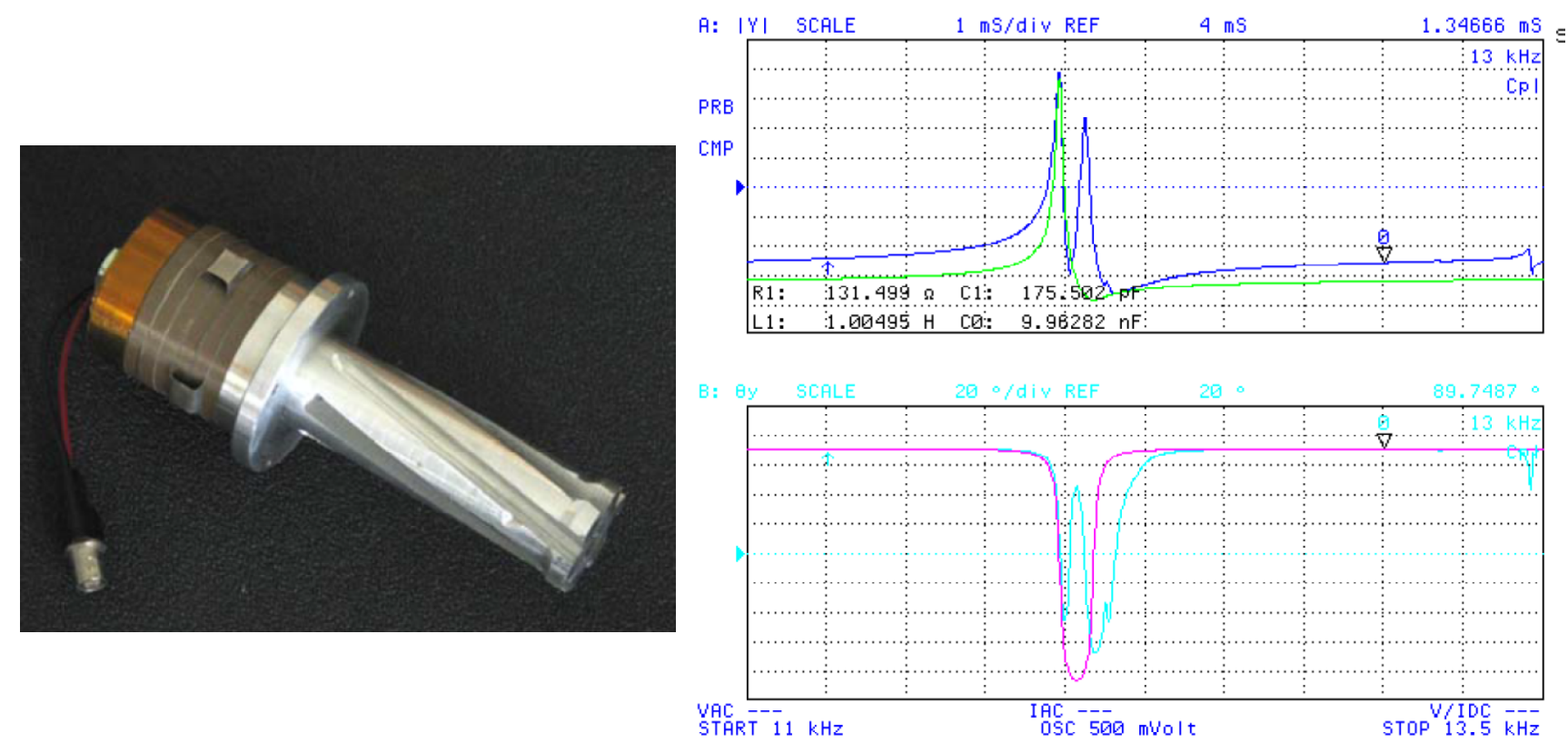

Figure 6. Photograph of the fabricated transducer. Six PZT ceramics are pre-stressed to $15 \mathrm{MPa}$ between the horn and backing using a pre-stress bolt and its admittance spectra (magnitude and phase angle) and a fit using Van Dykes Equivalent circuit.

The transducer is composed of a stainless steel backing, a stack of six PZT piezoelectric ceramics, and an aluminum horn with angled cuts. The angled cuts were made by mounting the horn at an angle in a mill, and milling straight across. The performance of the transducer was first analyzed using an impedance analyzer to obtain its admittance spectrum, which is shown above in Figure 6 along with the impedance analyzer equivalent circuit fit. The impedance analyzer uses parallel elements of a C-RLC circuit to model the transducer at resonance.

The double peak in the admittance spectrum is likely caused by an overlap between the axial mode and a bending or torsion mode. This double peak causes some error in the fit. Using the parallel C-RLC equivalent circuit fitting routine within the impedance analyzer, the approximate resonant frequency and the electromechanical coupling coefficient can be obtained. The results derived from the impedance analysis are shown in Table 2.

Table 2. Transducer performance derived from impedance analysis.

\begin{tabular}{|c|c|}
\hline First Resonant Frequency & Electromechanical Coupling Coefficient \\
\hline $11972 \mathrm{~Hz}$ & 0.07 \\
\hline
\end{tabular}

A Fotonic sensor was used to determine the actual tip displacement and tip rotation of the transducer for various input power levels. The Fotonic sensor is able to measure high frequency, low amplitude displacements using a light emitting/receiving fiber optic bundle. The measured tip displacement versus input power to the transducer is shown in Figure 7. 


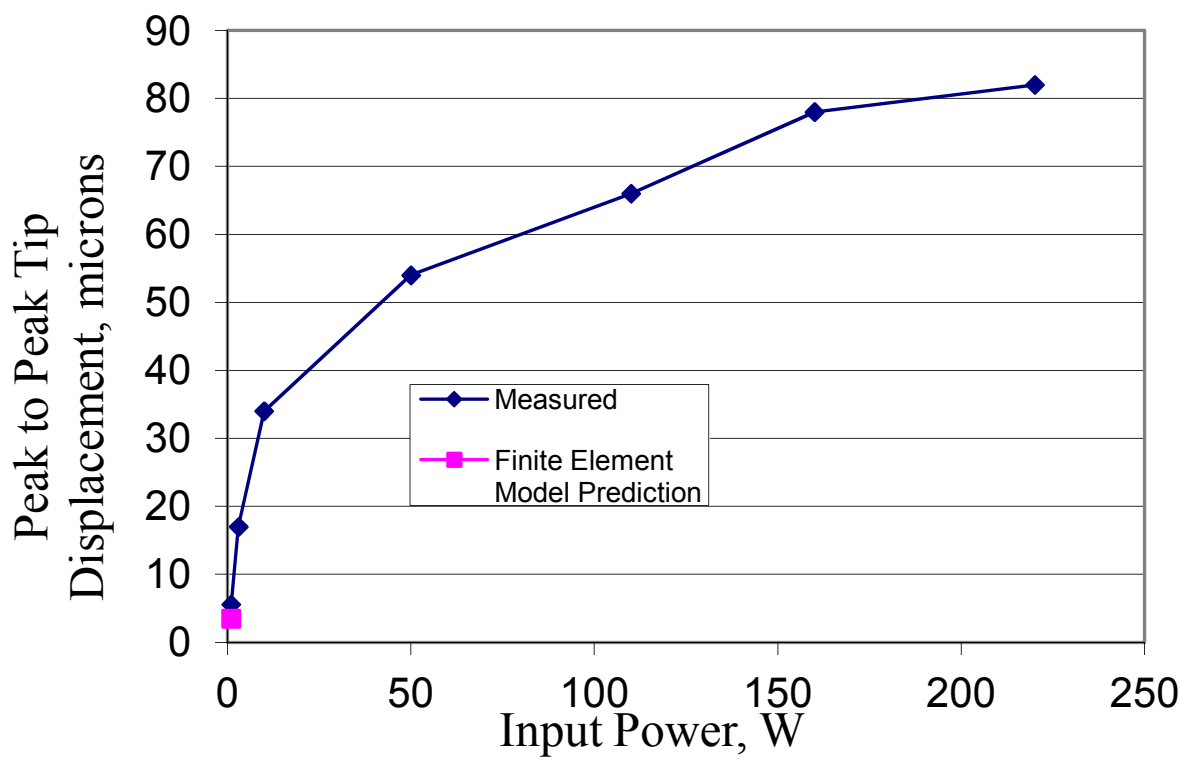

Figure 7. Horn tip displacement versus input power. Measurements taken using a Fotonic sensor, as well as the Finite Element model prediction. The Finite Element model was only analyzed for 1 Watt power input. Future modeling will examine higher power inputs in order for comparison to the measured data, however, there would likely be error in the prediction caused by non-linear effects not taken into account in the Finite Element model.

The Finite Element model predicted a horn tip displacement of $1.75 \mu \mathrm{m}$, and rotation of 4.4E-5 rad for $1 \mathrm{~W}$ power input. Extrapolating from the curve of the measured data, it appears reasonable to assume that the measured tip displacement at $1 \mathrm{~W}$ power input would be of the same order of magnitude as the model prediction. There are non-linear effects present in the transducer not taken into account in the Finite Element model which would likely cause some error between predicted and measured values. In order to measure the approximate tip rotation of the transducer, the probe was used to measure the displacement at a tangent to the horn's circular cross section. From this tangential displacement measurement, the tip rotation could be obtained using distance from the horn center. The experimental set-up of this measurement is shown in Figure 8 below.

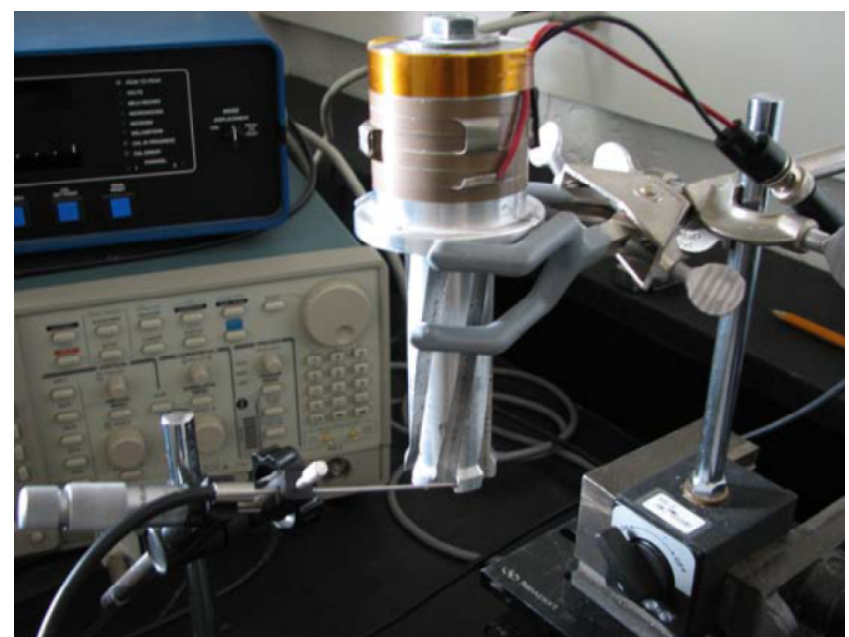

Figure 8. Experimental set-up for making tip rotation measurement. The probe is positioned at the horn tip to measure tangential displacement, from which rotation is calculated. The tip rotation derived from measuring tangential displacement versus input power is plotted in Figure 9. 


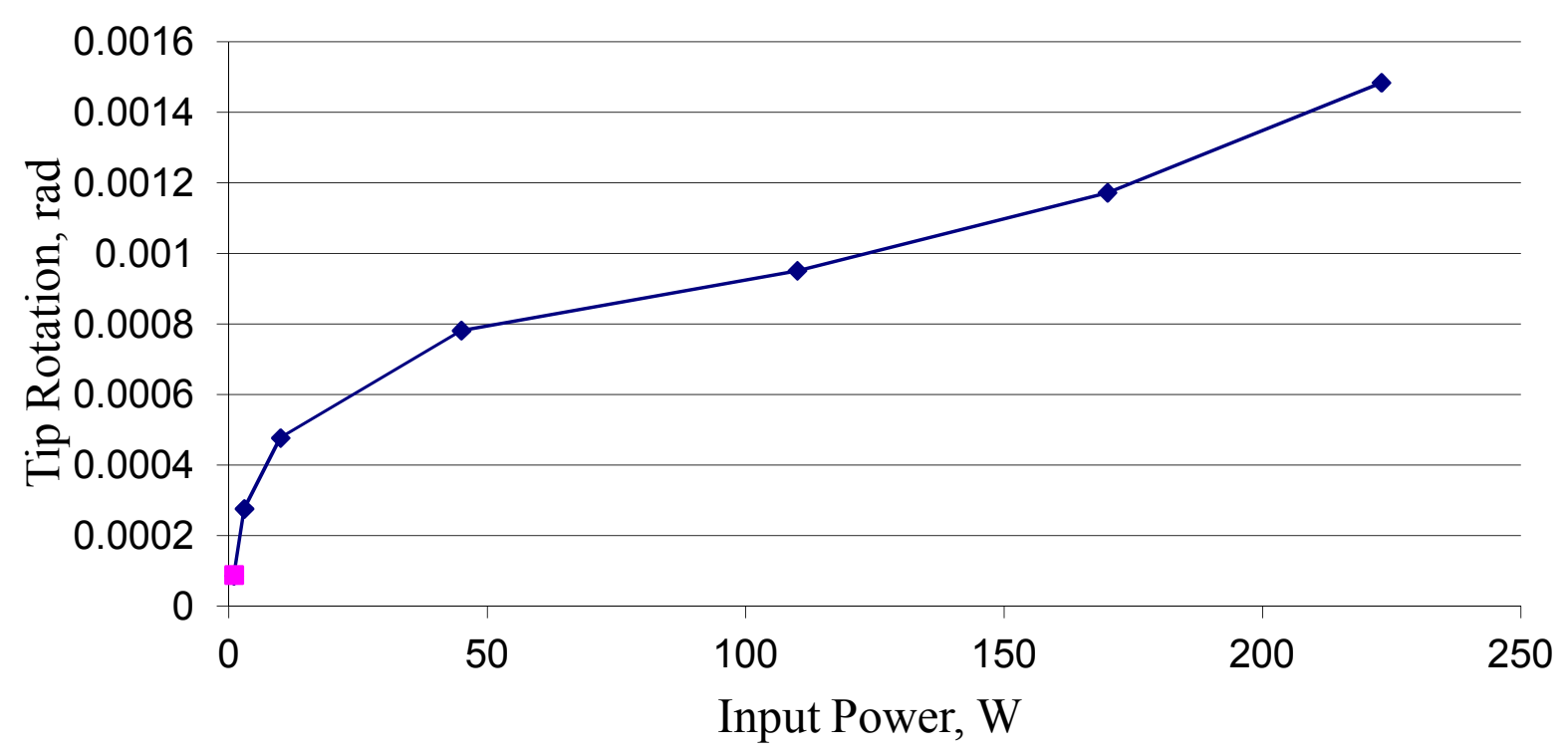

Figure 9. The Horn tip rotation versus input power. Measurements taken using a Fotonic sensor, The low power linear Finite Element model prediction is also shown (pink square). It is expected that at higher amplitudes there would likely be error in the prediction caused by non-linear effects not being taken into account in the Finite Element model.

As with the tip displacement, a direct comparison with the Finite Element Model tip rotation prediction was not made in Figure 9, however, the data seems to indicate that the model prediction and an extrapolation of the measured data are within the same order of magnitude. As well as to compare with the Finite Element model, the measured tip rotation was also used to predict the rotation speed that would be produced in the drill string. By multiplying the total tip rotation per cycle by the cycle frequency, an average bit rotation was estimated. The estimation assumes minimal to no slip occurring at the horn tip/free mass interface. The estimation predicts that for a power input of $110 \mathrm{~W}$, the drill bit will rotate at about $110 \mathrm{RPM}$, and that rotation speed increases with power input. The predicted drill bit rotation is compared to final drill testing results in the following section. A CAD model of the final transducer assembly is shown in Figure 10.

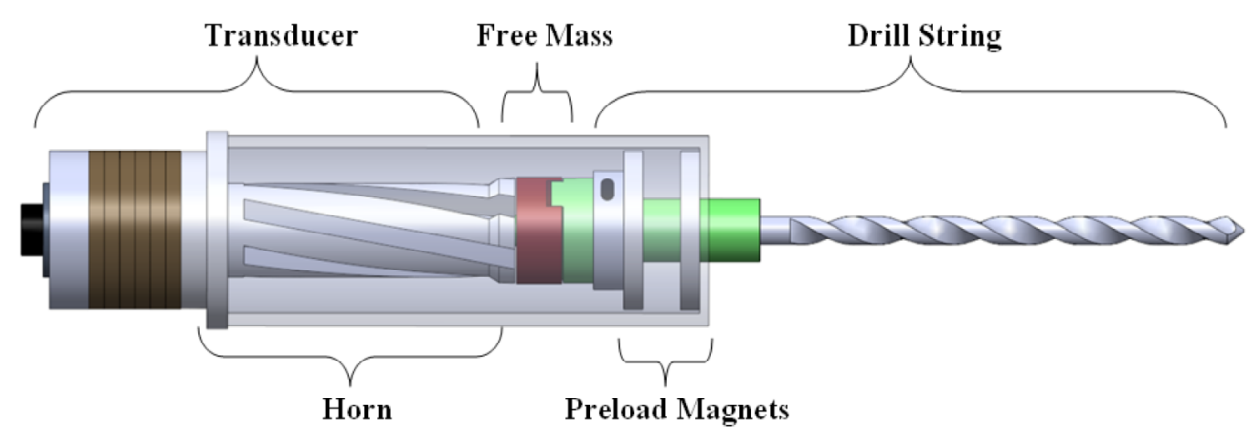

Figure 10. The CAD model of the final transducer assembly.

\section{B. Testing Results}

Once the transducer had been fabricated, the other components of the Piezoelectric Rotary Hammer Drill were machined and assembled. A test-bed was also set up in order to test the performance of the drill. A photograph of the drill and experimental test set-up is shown in inset photograph of the drill data shown in Figure 11. A function generator, power amplifier, oscilloscope, and PC were used to control the PRHD. A LabVIEW program was used to 
keep the drive frequency as close as possible to the transducer's resonant frequency, which tends to drop as the drill heats up. This HillClimb program ${ }^{23}$ functions by tracking the current entering the transducer and adjusting the input frequency such that the current is maximized (note that current is maximized at resonance). Initial testing of the Piezoelectric Rotary Hammer Drill has demonstrated consistent rotation and hammering present in the drill string. Driven at its first resonant frequency of about $12 \mathrm{kHz}$, the drill bit rotates counter clockwise (looking from the drill bit end). As mentioned before, a single piezoelectric actuator is used to drive both of these motions through the use of the torque inducing horn. One particular area of interest in the performance of the Piezoelectric Rotary Hammer Drill is the bit rotation produced for a given input power. Preliminary results of the bit rotation speed for various input power and operating conditions are shown in Figure 11. The plot includes a comparison with the rotation speed predicted by the horn tip rotation measurement mentioned above.
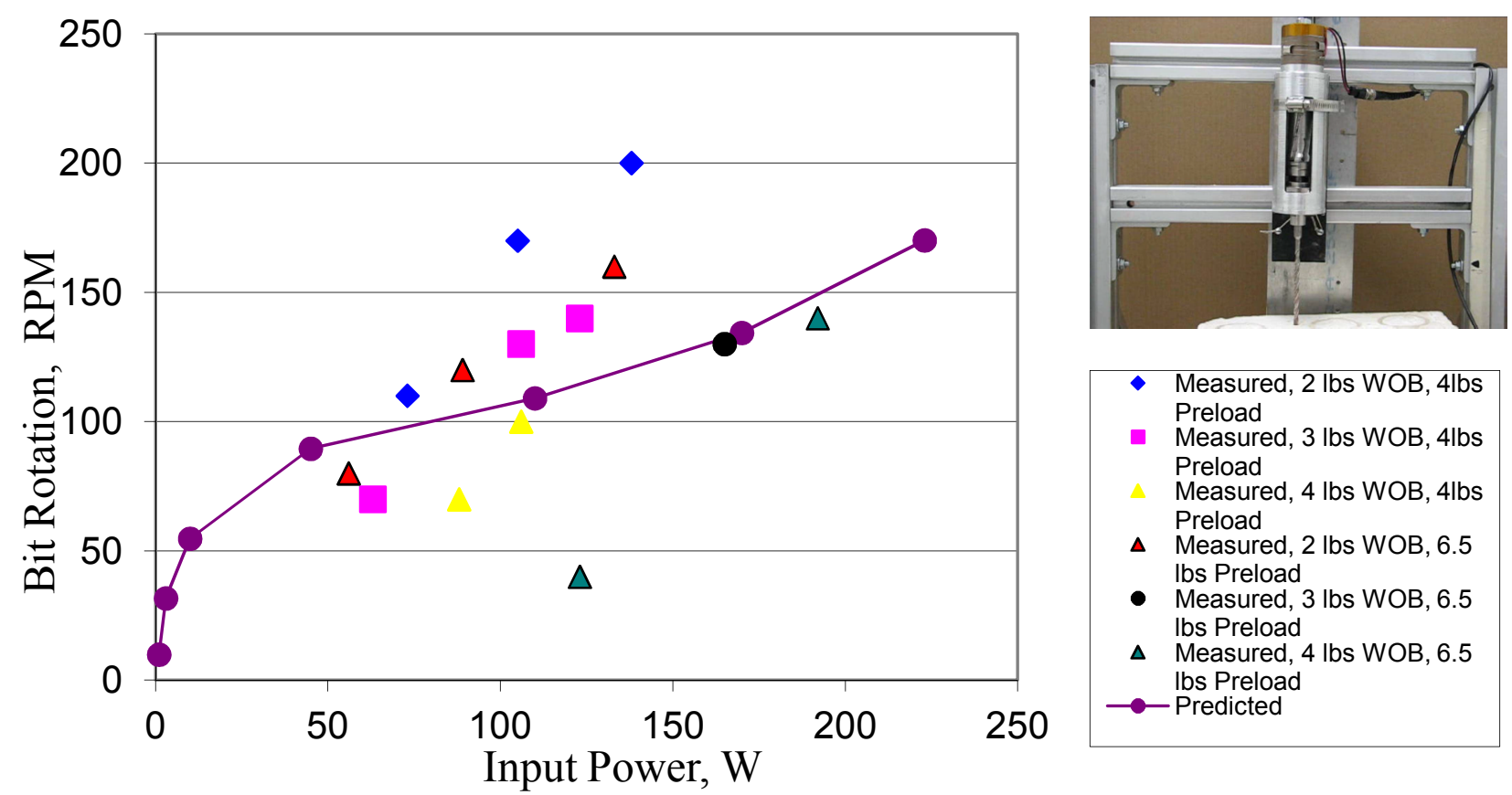

- Measured, 2 lbs WOB, 4lbs Preload

- Measured, $3 \mathrm{lbs}$ WOB, 4lbs Preload Measured, $4 \mathrm{lbs}$ WOB, $4 \mathrm{lbs}$ Preload

$\Delta \quad$ Measured, $2 \mathrm{lbs}$ WOB, 6.5 lbs Preload

- Measured, 3 lbs WOB, 6.5 Ibs Preload

$\Delta \quad$ Measured, $4 \mathrm{lbs}$ WOB, 6.5 Ibs Preload —-Predicted

Figure 11. Predicted and measured drill bit rotation speed. The drill was tested at various operating conditions. WOB represents the weight-on-bit, or the force acting on the drill bit end. The preload is the force from the repulsive magnets loading the drill string and free mass against the horn tip. An estimation of bit rotation is also predicted using the measured horn tip rotation, assuming no slip between the horn tip and free mass. The test setup is shown in the photograph.

The Piezoelectric Rotary Hammer Drill demonstrates a bit rotation of about $100 \mathrm{rpm}$ for about $100 \mathrm{~W}$ input power. The measured bit rotation shown above fits reasonably well to that predicted by the tip rotation measurement extrapolated from data determined using the Fotonic sensor. Errors may come from the fact that successive impacts between the free mass and horn tip likely occur at different instances of the horn tip's extension/retraction cycle. Depending on the instant of impact, the horn tip may not be rotating at the same speed. Also, it should be noted that as WOB and preload increase, the bit rotation speed decreases for a given input power. The extra forces loading the drill string and free mass against the horn likely cause some slipping between the free mass and horn tip. All of the data indicates that bit rotation speed increases with input power, however, more work is needed to fully characterize this relationship. The results presented here represent only preliminary tests of the prototype drilling capabilities. The best drilling rates that have been achieved are shown in the table below. These results were obtained drilling for 10 minutes into limestone. Note that the duty cycle represents the percentage of time that the drill is turned on. A duty cycle of $50 \%$ 
could represent the drill being alternately on for 3 seconds, and off for 3 seconds. Duty cycling is necessary to reduce overheating in the prototype drill when driving at high power levels.

Table 3. Best drilling achieved using initial prototype.

\begin{tabular}{|c|c|c|c|c|}
\hline Input Power, W & WOB, lbs & Preload, lbs & Duty Cycle & Drill Rate, $\mathbf{m m} / \mathbf{m i n}$ \\
\hline 100 & 3 & 4 & $80 \%$ & 8 \\
\hline
\end{tabular}

As mentioned previously, the drill prototype is un-optimized, and higher drilling rates can be expected in future designs. Simply by decreasing the free mass weight by $25 \%$, the drill rate was increased from 2 to $8 \mathrm{~mm} / \mathrm{min}$. Another test result worth noting is that rotation in the opposite direction (clockwise) could be produced when the transducer was driven at its second resonance frequency at about $22.4 \mathrm{kHz}$. At this frequency minimal extension and hammering action is present and therefore the "drill" could possibly be designed to act as a pure rotary motor. A transducer which can produce linear actuation and rotary actuation in two directions could prove to be a promising tool on future NASA missions. In circumstances where both a linear and rotary actuator would typically be required, they could be replaced by a single actuator.

\section{CONCLUSIONS}

A novel concept to produce hammering and rotation using a single piezoelectric transducer was investigated theoretically using a variety of modeling methods via MATLAB and ANSYS. A transducer design was chosen for fabrication which exhibited both rotation and axial extension at the horn tip at resonance. The fabricated prototype has demonstrated that simultaneous rotation and hammering can be produced in a drill string through the use of a single piezoelectric actuator. The ability to produce rotary-hammer drilling by means of a single actuator offers the potential for low complexity, low mass and low power sampling tools that could be designed for future NASA sampling missions. This Piezoelectric Rotary Hammer Drill prototype has not been optimized, and requires further development. Future work should include considering different horn shape designs, in order to compare with the performance of this first prototype. In addition looking at other geometries and horn materials such as Titanium may aid in increasing the electromechanical mechanical coupling. The harder contact surface of a Titanium horn tip may also provide better hammering against the free mass. In fabricating this prototype, the selection of a free mass size has been somewhat arbitrary, though efforts have been made to keep it proportional to the free mass sizes of past drills developed at the JPL NDEAA lab. Testing of multiple free mass sizes will help to optimize the drills performance. Future work should also explore the ability of the drill to reverse rotation direction when driven at different frequencies. A single transducer which can produce linear and rotary actuation in two directions would increase the utility of tools designed based on this effect.

\section{ACKNOWLEDGMENTS}

This research was carried out at the Jet Propulsion Laboratory, California Institute of Technology, and was sponsored by National Aeronautics and Space Administration (NASA). Reference herein to any specific commercial product, process, or service by trade name, trademark, manufacturer, or otherwise, does not constitute or imply its endorsement by the United States Government or the Jet Propulsion Laboratory, California Institute of Technology.

\section{REFERENCES}

\footnotetext{
${ }^{1}$ S. Sherrit, B.P. Dolgin, Y. Bar-Cohen, D. Pal, J. Kroh, T. Peterson "Modeling of Horns for Sonic/Ultrasonic Applications", Proceedings of the IEEE Ultrasonics Symposium, pp. 647- 651, Lake Tahoe, Oct 1999

${ }^{2}$ Y. Bar-Cohen, S. Sherrit, B.P. Dolgin, T.M. Peterson, D. Pal and J. Kroh, R. Krahe, "Smart Ultrasonic Driller Corer", Patent \# $6,863,136$

${ }^{3}$ Benjamin Dolgin, Stewart Sherrit, Yoseph Bar-Cohen, Richard Rainen, Steve Askin, Donald Bickler, Donald Lewis, John Carson, Stephen Dawson, Xiaoqi Bao, Zensheu Chang, Thomas Peterson Ultrasonically Actuated Tools for Abrading Rock Surfaces" (NPO30403), NASA Tech Briefs, Vol. 30, No. 7, pp. 58, July, 2006
} 
${ }^{4}$ S. Sherrit Y. Bar-Cohen, X. Bao , Z. Chang, and D. Blake, C. Bryson "Powder Collection System for the Ultrasonic/Sonic Driller/Corer" NASA Tech Brief, NPO-40564, 29,1, pp. 51, Jan 2005

${ }^{5}$ B. Dolgin, S. Sherrit, Y. Bar-Cohen, S. Askins, D. Sigel, X. Bao, and Z. Chang, "Ultrasonic/ Sonic Vibrating/Rotating Tool" NASA Tech Brief, NPO-30370, 26, 9, pp.65, Sept 2002

${ }^{6}$ Mircea Badescu, Steven Kassab, Stewart Sherrit, Jack Aldrich, Xiaoqi Bao, Yoseph Bar-Cohen, and Zensheu Chang, "Ultrasonic/Sonic Driller/Corer as a hammer-rotary drill," Proceedings of the SPIE 14th International Symposium on Smart Structures and Materials, San Diego, CA, SPIE Vol. 6529-26, 18-22 March, 2007.

${ }^{7}$ Maurer W. C., "Novel Drilling Techniques," Pergamon Press, New York, (1968)

${ }^{8}$ Bar-Cohen Y., and K. Zacny (Eds.), Drilling in Extreme Environments - Penetration and Sampling on Earth and Other Planets, Wiley - VCH, Hoboken, NJ, ISBN-10: 3527408525, ISBN-13: 9783527408528, (July 2009).

${ }^{9}$ S. Sherrit, X. Bao, M. Badescu, Y. Bar-Cohen, "Single Piezo-Actuator Rotary-Hammering (SPARH) Drill”,NASA New Technology Reprot Filed July 21, 2009, Provisional Patent Application Ser. No. 61/240,569 filed Sep. 8, 2009, US Patent application \#20110056713, filed Sept 8, 2010.

${ }^{10}$ L. Domm, S. Sherrit, and Y. Bar-Cohen, "Development of a Piezoelectric Rotary Hammer Drill," NASA USRP - Internship Final Report, 2011. http://usrp.usra.edu/technicalPapers/jpl/DommMay11.pdf

${ }^{11}$ Harkness, P., Cardoni, A., Lucas, M., and Waugh, L., "A Simple, Lightweight and Low-Reaction Deployable Architecture for Subsurface Sample Retrieval,” AIAA Space Sept, 14-17, 2009, Pasadena, USA, 2009. AIAA 2009-6507.

${ }^{12}$ Patrick Harkness, Andrew Mathieson, Christopher Murray and Margaret Lucas,"Optimization of Ultrasonic Horns for Momentum Transfer and Survivability in High-Frequency/Low Frequency Planetary Drill Tools" AIAA Space Sept. 27-29, 2011, Long Beach, USA, AIAA 2011-7319

${ }^{13}$ Sashida T., Kenjo T (1993): An Introduction to Ultrasonic Motors, Claredon Press , Oxford

${ }^{14}$ Ueha S., Tomikawa Y.( 1993): Ultrasonic Motors, Claredon Press, Oxford

${ }^{15}$ Uchino K. (1996): Piezolectric Actuators and Ultrasonic Motors (Electronic Materials--Science \& Technology, 1), Kluwer Academic Pub; ISBN: 0792398114

${ }^{16}$ S. Sherrit, B.P. Dolgin, Y. Bar-Cohen, D. Pal, J. Kroh, T. Peterson "Modeling of Horns for Sonic/Ultrasonic Applications", Proceedings of the IEEE Ultrasonics Symposium, pp. 647- 651, Lake Tahoe, Oct 1999.

${ }^{17}$ S. Sherrit, X. Bao, Z. Chang, B.P. Dolgin, Y. Bar-Cohen, D. Pal, J. Kroh, and T. Peterson, "Modeling of the Ultrasonic/Sonic Driller/Corer: USDC," Proceedings of the IEEE Ultrasonics Symposium, pp. 691-694, San Juan, Puerto Rico, Oct 22-25, 2000.

${ }^{18}$ Y. Bar-Cohen, S. Sherrit, B. Dolgin, D. Pal, T. Peterson J. Kroh, and R. Krahe, "Ultrasonic/sonic drilling/coring (USDC) for in-situ planetary applications," Proceedings of the SPIE Smart Structures Conference, held at Newport Beach, CA., March 2000, SPIE, Vol. 3992, Paper No. 101, 2000.

${ }^{19}$ X. Bao, Y. Bar-Cohen, Z. Cheng, B.P. Dolgin, S. Sherrit, D.S. Pal, S. Du, T. Peterson, "Modeling and Computer Simulation of the Ultrasonic/Sonic Driller/Corer (USDC)", IEEE Transactions on Ultrasonics, Ferroelectrics and Frequency Control, 50, pp. 1147-1160, Sept. 2003.

${ }^{20}$ D.F. Blake, P. Sarrazin, S. J. Chipera, D. L. Bish, D. T. Vaniman, Y. Bar-Cohen, S. Sherrit, S. Collins, B. Boyer, C. Bryson and J. King, "Definitive Mineralogical Analysis of Martian Rocks and Soil Using the CHEMIN XRD/XRF Instrument," Proceedings of the Sixth International Conference on Mars, held at Caltech, Pasadena, CA, July 20-25, 2003.

${ }^{21}$ Stewart Sherrit, Xiaoqi Bao, Mircea Badescu, , Phillip Allen, Yoseph Bar-Cohen, "Monolithic Rapid Prototyped Flexured Ultrasonic Horns", Proceedings of the IEEE International Ultrasonics Symposium, pp. 886-889, Presented in San Diego, CA, October 2010.

${ }^{22}$ Kumada, A. (1985): "A Piezoelectric Ultrasonic Motor," Japanese Journal of Applied Physics, Vol. 24, Supplement 24-2, pp. 739-741

${ }^{23}$ Jack Aldrich, Stewart Sherrit, Xiaoqi Bao, Yoseph Bar-Cohen, Mircea Badescu, and Zensheu Chang, "Extremum-seeking control for an ultrasonic/sonic driller/corer (USDC) driven at high power", Proc. SPIE 6166, 6166-18 (2006) 